\title{
Téoros
}

Revue de recherche en tourisme

\section{Clientèles touristiques}

\section{L'avenir est à la quête de l'exotisme}

\section{Laurent Bourdeau et Pascale Marcotte}

Volume 18, numéro 3, automne 1999

Le tourisme dans tous ses états

URI : https://id.erudit.org/iderudit/1071849ar

DOI : https://doi.org/10.7202/1071849ar

Aller au sommaire du numéro

Éditeur(s)

Université du Québec à Montréal

ISSN

0712-8657 (imprimé)

1923-2705 (numérique)

Découvrir la revue

Citer cet article

Bourdeau, L. \& Marcotte, P. (1999). Clientèles touristiques : l'avenir est à la quête de l'exotisme. Téoros, 18(3), 29-32. https://doi.org/10.7202/1071849ar d'utilisation que vous pouvez consulter en ligne.

https://apropos.erudit.org/fr/usagers/politique-dutilisation/ 


\section{A : : : : : : : : : : : : : : : : :}

\section{Clientèles touristiques}

\section{L'AVENIR EST A LA QUĖTE DE L'EXOTISME}

\section{Laurent Bourdeau et Pascale Marcotte}

Plusieurs obsenvateurs affirment que le nombre de touristes, tant d' affaires que d'agrément, augmentera au cours des prochaines années. Cette annonce représente la possibilité de commercialiser moult services touristiques pour répondre à la demande touristique sous la forme du tourisme de masse. On constate d'ailleurs l'apparition de nouvelles destinations et de nouveaux services touristiques, tant sur le plan du tourisme réceptif que du tourisme international.

À court et à moyen terme, cette sollicitation des clientèles d'affaires et d'agrément devrait s'accroitre non seulement par le lancement de nouveaux services touristiques, mais également par une transformation du réseau de distribution (vente par Internet et par télévision interactive). Bref, les propositions de voyages associés au tourisme se font de plus en plus nombreuses pour répondre à la demande des individus.

Ces propositions de voyages semblent justifiées si l'on considère l'augmentation de voyageurs. Toutefois, au XXI' siècle, la croissance de propositions de voyages associés au tourisme de masse et standardisés ne garantira pas une adhésion inconditionnelle des individus au monde du voyage, Gourant (1993) a d'ailleurs soulevé cette question dans un essai prospectif portant sur le tourisme international. Il annonçait qu'à l'aube de l'an 2000, un des défis de l'industrie touristique serait d'éviter la dégradation du produit touristique par le tourisme de masse. Depuis la parution de cet article, on constate, comme le prévoyait l'auteur, une concentration des acteurs de l'industrie touristique et l'accroissement continu du tourisme de masse.
Jusqu'ici, cette concentration du réseau de distribution et des producteurs touristiques a favorisé l'offre d'un plus grand nombre de services touristiques de plus en plus associés au divertissement et à la création de lieux artificiels. En bref, moins d'entreprises offrent un plus grand nombre de services. L'industrie oxygène alors le tourisme par la création d'un plus grand nombre de services et de destinations touristiques.

Quand on s'intêresse aux comportements de voyage futur des individus, on ne peut que s'intéresser aux motivations de voyage, éléments déclencheurs et préalables à l'acte d'achat. À première vue, devant l'augmentation du nombre de touristes annoncée au cours des prochaines années et devant l'accroissement des services touristiques, il semble que les voyageurs se feront plus nombreux au cours du XXI's siècle. Cependant, en considérant l'évolution des motivations des voyageurs, ont peu s'interroger sur les intentions futures en matière de voyage. En d'autres mots, on peut se demander si les individus manifesteront toujours le même degré de motivations face au tourisme.

\section{LES MOTIVATIONS À VOYAGER}

De nombreuses recherches ont identifié les raisons pour lesquelles les touristes achètent un voyage. Bien que des différences existent entre le segment du tourisme d'affaires et celui du tourisme d'agrément, le rapport qualité - prix jone un rôle majeur dans la prise de décision. En d'autres mots, le touriste ne recherche pas nécessairement le service le moins cher. Pourtant, de nombreux acteurs de l'industrie touristique se livrent une guerre de prix dont la stratégie consiste à influencer la décision d'achat uniquement en fonction du prix le plus bas. Le tourisme de masse se développe alors à partir d'une guerre de prix sans que les acteurs de l'industrie touristique ne portent une grande attention aux motivations de voyage, eléments préalables au comportement d'achat. On offre alors un objet de consommation sans nécessairement comprendre les motivations qui poussent les individus à interagir avec cet objet.

En cette fin de $X X^{e}$ siècle, un nombre important d'individus se situent dans une * ère de divertissement * touristique'. Les motivations de ces touristes sont alors associées à la capacité de divertissement offerte par le lieu touristique, au fait de fuir le quotidien (Mannell et Iso-Athola, 1987) ou d'impressionner l'entourage ${ }^{2}$. Par contre, d'autres individus se trouvent encore dans une « ère de découverte * touristique. L'attrait du lieu visité, de l'authenticité des lieux et du contact avec le peuple d'une région exotique, compose toujours leurs motivations au voyage. Par la visite d'attractions touristiques, le touriste tente alors de découvrir les caracté- 
ristiques de la société, son héritage patrimonial et son identité culturelle ${ }^{3}$.

En observant les divers services touristiqués destinés au tourisme d'agrément, on constate donc que les touristes voyagent pour apprendre et entrer en contact avec d'autres cultures ou pour le simple plaisir de se divertir, de rêver, peu importe le lieu visité. Les motivations des touristes se basent alors sur lạ quête d'exotisme à l'étranger. En voyageant, les individus peuvent entrer en contacter avec a l'étrangeté * loin de leur résidence habituelle.

Toutefois, on constate que d'autres individus choisissent de ne pas voyager, parce qu'ils peuvent rencontrer cette «étrangetés tout en restant bien installés chez eux. Ils peuvent alors entrer en contact avec des éléments qui leur permettent de vivre une experience différente dans leur propre milieu de vie. Les motivations de ces individus sont alors liées à une quête de l'exotisme chez soi'.

L'analyse des motivations associées au contenu des voyages d'agrément permet de penser qu'au cours des prochaines années, une proportion importante d'individus sera toujours soit dans une * ère de divertissement $*$ soit de « découverte $*$ touristique. Toutefois, si traditionnellement le voyage, et principalement les voyages internationaux, répondaient aux motivations des individus basées sur la quête d'exotisme à l'étranger, les tendances sociales futures pourraient fournir d'autres choix pour répondre à ces motivations.

\section{LES TENDANCES SOCIALES ET LE COMPORTEMENT DE VOYAGE}

Actuellement, l'expérience de l'exotisme permet à l'individu de se differencier des autres par la visite de pays étrangers, par ce qui a été vu et vecu. Alors que la rếponse a la motivation de vivre du « différent résidait traditionnellement dans le voyage, le $\mathrm{XXI}^{e}$ siècle fournira de plus en plus aux individus, qu'ils voyagent ou non, la possibilité d'assouvir cette quette d'exotisme à l'aide d'autres objets de consommation.

Les individus, touristes ou non, ont maintenant la possibilité d'entrer en contact avec le caractère exotique des sociétés gräce aux différents outils de communication, et ce même si ce contact semble artificiel ou virtuel. En effet, ceux qui cherchent encore à connaître d'autres cultures pourront assouvir une part de leur quête par les nouvelles technologies en voyageant en peu de temps, partout, et à peu de frais. Cependant, si a jadis $x$ la télévision a augmenté l'intếrêt des individus par rapport aux voyages et aiguisé la curiosité des plus sédentaires, les nouvelles technologies pourtont également banaliser l'exotisme et l'étrangeté et, par conséquent, la motivation à voyager. Les nouvelles technologies semblent ainsi jouer un rôle contradictoire qui freine la motivation au voyage. En fait, les individus auront toujours le besoin et l'envie de rever, mais l'objet de consommation qu'est le voyage ne sera plus la seule grande réponse au rêve et à la quête d'exotisme. On passera done de l'étrangeté à l'exotisme chez soi.

On peut également suggérer que le pouvoir d'attraction des lieux touristiques sera frappé par le même phénomène qui accable le milieu de l'art. Les lieux touristiques perdront leur a aura (Benjamin, 1971). En effet, avec les modes de reproduction technique, le fait de voir et d'avoir accès à l'ceuvre d'art diminue l'attrait de l'original. L'individu peut se contenter d'une reproduction ou d'une image. Tout comme une ouvre d'art, 1'attrait du véritable lieu touristique pourrait diminuer aux yeux du touriste. Bref, le lieu touristique perdra de son pouvoir d'attraction par sa représentation de plus en plus accessible grâce aux divers outils de communication et de consommation. On risque donc d'assister, au cours des prochaines années, à une désacralisation du véritable lieu touristique au détriment d'une sacralisation de la représentation de l'attraction touristique et ce, parce qu'il sera de plus en plus facile de vivre l'exotisme chez soi.

$\mathrm{Si}$, dans certains lieux touristiques, le tourisme a permis de répondre à un certain nombre de motivations de nature culturelle et de divertissement, il a également reçu les mêmes critiques que celles formulées à l'egard de la culture de masse, soit la facilité, l'absence d'effort, l'uniformité, l'appauvrissement culturel, 1'abrutissement et l'aliénation de l'individu ${ }^{5}$. II n'est d'aucun secret que le tourisme implique une uniformisation de la prestation touristique et, éventuellement, une standardisation (Gourant, 1993). En fait, le tourisme a, dans certains lieux, détruit le * produit $*$ touristique. Le $\alpha$ different $*$ qui motivait le voyage est éclipsé au profit d'un produit précconçu, un produit que l'on pourrait qualifier de $\alpha$ non-lieu $*^{6}$ ou d'artificiel.

Le développement touristique uniquement conçu avec l' idée de la rentabilité a entraîné la détérioration des relations et des perceptions des touristes et des hôtes. Avec la mondialisation, le tourisme standardisé devient de plus en plus commun et de moins en moins attrayant. Si l'espace géographique conditionne le paysage, le service touristique et ses infrastructures peuvent facilement être copiés et peuvent donc se retrouver partout.

Alors qu' a la fin du $\mathrm{XX}^{\mathrm{c}}$ siècle les individus évoluaient dans une société offrant certaines périodes de loisirs et de temps libre, l'organisation du travail au $\mathrm{XXI}^{e}$ siècle tendra à réduire le temps consacré au voyage. En effet, les changements observés dans l'organisation du travail entraînent des modifications des comportements liés au voyage. Les individus auront soit beaucoup de temps libre et peu d'argent à consacrer au voyage, soit assez d'argent pour voyager, mais peu de temps libre. En fait, il y aura un nombre grandissant d' individus à la retraite, de travailleurs autonomes et d'étudiants (les études se poursuivant plus longtemps) et les écarts de revenus entre riches et pauvres iront en s'accentuant. Si le nombre de voyages se maintient, ils seront de courte durée et se feront sur de petites distances. Les voyages de longue durée étant trop chers pour ceux dont les revenus sont incertains ou diminuếs, l'exotisme chez soi prendra une plus. grande importance.

Le risque associé à lạ décision en matière de consommation de produit touristique sera également accru. On peut penser qu'avec la concentration des richesses, même si le voyage n'est plus une activité de luxe, il se peut fort bien que cet objet de consommation redevienne une activité d'élite, réservée aux individus qui ont les moyens de payer et qui ont le temps de vivre cette expérience exotique.

Devant l'absence de temps à consacrer au voyage et $1^{\prime}$ incertitude des revenus, le tourisme réceptif pourrait se développer. Toutefois, un tourisme réceptif standardisé et identique aux \& produits $*$ touristiques internationaux, artificiels et homogènes, aura une duré de vie très courte. Les gens. chercheront plutôt une forme de tourisme authentique. Les touristes à la recherche 
d'une forme d'exotisme tenteront de sortir des * sentiers battus * de l'industrie pour s'aventurer auprès d'entreprises touristiques qui proposent une forme de tourisme réceptif plus sensuel et proche du \& véritable $*$. Les voyageurs ne voudront plus se voir associes aux consommateurs insouciants, pollueurs et abrutis, téputation qui a longtemps suivi le touriste. Ils chercheront davantage a \& s'impliquer socialement $\%$. Les clientèles chercheront à faire preuve a d'éthique dans la consommation de l'objet touristique. De plus, ces clientèles cherchant à vivre une expérience authentique consommeront cet objet touristique, même si cette expérience est située près de leur lieu de résidence. En conséquence, l'absence d'argent et de temps à consacrer au voyage augmentera le nombre d'excursionnistes.

L'insécurité physique et psychologique affectera aussi les motivations à voyager. L'insécurité perçue par le touriste qui voyage dans plusieurs des grandes villes abritant des attractions touristiques importantes accentuera la quête de l'exotisme chez soi. En effet, pourquoi le touriste se rendrait-il dans une grande ville où il craint de sortir de sa chambre d'hôtel en soirée, où il doit éviter scrupuleusement certains quartiers et cacher sa caméra vidéo pour camoufler son statut?

Si l'on tient compte de ces tendances, nous assisterons à ce que nous appelons \& l'exotisme chez soi $\%$. En effet, pourquoi partir en voyage lorsque \& l'étrangeté $*$ et l'exotisme se retrouvent chez soi ? Pourquoi l'individu se déplacerait-il dans un autre lieu alors que le monde devient commun et quotidien ? Les touristes trouveront une motivation à voyager par les services et les a produits « touristiques qui leur permettront de vivre quelque chose de différent, tout en tenant compte des contraintes de temps et de revenus. De façon générale, les gens qui voyageront le feront pour donner un sens à leur vie et ils rechercheront des relations authentiques. Les grandes attractions touristiques artificielles poursuivront leur croissance dans la mesure où elles permettront aux touristes de ressentir de fortes émotions, en toute sécurité, dans un milieu de visite différent.

\section{L'EFFET DE L'EXPÉRIENCE ANTÉRIEURE SUR LE TOURISME}

L'expérience accumulée en matière de voyage par le touriste affectera également son comportement de voyage futur. Il est à prévoir qu'il sera difficile de faire k sortir $\times$ un individu de sa maison et de le transformer en touriste. En fait, les touristes ayant beaucoup d'expériences antérieures risquent de perdre le goût de la decouverte à cause de la standardisation, de l'uniformisation et de l'insécurité. L'accroissement de l'expérience des individus en matière de voyages risque donc d'insensibiliser le voyageur. Le degré d'expérience accumulé provoquera une perte de $\propto$ l'aura $\otimes$ du voyage. Qu'ils voyagent par choix ou par obligation, les individus feront des courts voyages et il sera difficile, dans le contexte du tourisme standardisé, d'amener les individus à voyager. En bref, le voyage d'agrément, dans le cadre du tourisme standardisé, sera vu comme un objet monotone. Dans ce contexte, le sentiment d'une dégradation du divers $\%$, comme le a jour où l'on redécouvrit que la terre est ronde $»$ risque de motiver le voyageur à rester chez lui (Segalen, 1986).

Dans leur analyse du comportement du touriste, les dirigeants des entreprises touristiques auront intérêt à ne pas mélanger motivations et processus d'achat du touriste. Avant de lancer de nouvelles destinations et de nouveaux services touristiques, les entreprises devront porter une attention particulière aux motivations de la clientèle et au degré d'expérience des touristes.

Les dernières années démontrent que les motivations des touristes ne sont pas toujours prises en compte par une industrie très standardisée, qui se base sur la quantité de services et de produits offerts, sans tenir compte de l'expérience touristique. Cette standardisation qui ne tient pas compte des motivations et des influences sociales ne sera pas un élément d'incitation au voyage. La consommation de l'objet touristique standardisé fera disparaître l'objet du désir, le voyage et le rêve. Avant de s"intéresser au comportement d'achat, il sera de plus en plus important de comprendre pourquoi les individus voyagent ou ne voyagent pas.

\section{CONCLUSION}

Au cours des prochaines années, les individus voyageront-ils? La réponse à cette question est positive pour les individus qui auront temps et argent. Cependant, la forme du voyage et de l'expérience permettant de réaliser le rêve pourrait changer.
Ce changement sera tributaire des motivations en matière de voyage. La quête de l'étrangeté et de l'exotisme chez soi pourrait accroitre la tendance ver's les courts séjours. La standardisation des produits touristiques, l'uniformisation et l'insécurité des grandes villes créeront un effet d'amplitude sur cette tendance aux courts séjours, mais aussi à voyager chez soi. On peut ajouter qu'il sera difficile d'influencer l'individu et de lui démontrer que la destination et le service touristiques permettent de réaliser ses rêves et d'assouvir' ses motivations.

Le tourisme standardise et de plus en plus homogène réduira l'intention de voyage. En conséquence, l'individu envisagera de plus en plus d'autres solutions, externes au monde du voyage, pour assouvir ses motivations. Le tourisme standardisé conduira done les touristes à considerer des alternatives au voyage. Quand les individus auront besoin de s'évader ou de « s'éprouver $s$, ils choisiront entre l'achat d'un voyage ou d'une piscine, ou encore entre l'achat d'un voyage ou d'un nouvel ordinateur. Tant qu'à trouver ailleurs la même chose que chez soi, le touriste préférera voyager en utilisant les nouvelles technologies et le confort de son foyer, tout en cherchant à se procurer un objet de consommation differrent de ceux qu'il possède dejà. Bref, il n'ira pas ailleurs s'il peut trouver la même chose chę lui. Le voyage ne sera plus le seul objet de consommation qui permettrạ la quête de la différénce. Le voyage, comme besoin d'évasion et de fuite, que les motivations soient de nature culturelle ou de divertissement, aura toujours comme exigence l'offre d'un caractère exotique et authentique.

Les lieux artificiels, souvent bâtis sur la spéculation, où personne ne réside en permanence, existeront encore au $\mathrm{XXI}^{e}$ siècle, mais deviendront des lieux fragiles dont le pouvoir d'attraction s'effritera rapidement. Dans ce genre de lieux artificiels, il sera difficile de faire vivre une expérience à un individu qui a beaucoup d'expérience en matière de voyage. L'effort consacré par l'individu pour sortir de chez lui risque de s'accroître au cours des prochaines années et il faudra alor's que le voyage offre davantage en compensation.

Dans les années à venir, les gens voudront vivre une expérience touristique esthétiquè, d'une grande beauté, qui leur apportera une satisfaction intếrieure et permettra 
de donner un sens à leur vie. Le touriste ne voudra plus avoir l'impression d'être touriste, mais cherchera une expérience touristique originale lui permettant d'assouvir sa quête d'exotisme. Le tourisme de masse artificiel et standardisé vivra un déclin. Les touristes rechercheront donc des produits différenciés et authentiques.

Devant ce constat, les entreprises touristiques doivent réorienter leurs stratégies de commercialisation afin de répondre aux motivations des touristes. Dans un premier temps, les entreprises touristiques doivent se différencier tant par la forme que par le contenu de l'expérience touristique. L'offre touristique devra donc se diversifier afin de répondre aux motivations d'exotisme et d'étrangeté recherchés par les touristes. Dans un deuxième temps, la différenciation de l'offre touristique doit se faire en tenant compte des autres produits ou services complementaires permettant de répondre aux besoins de rêver des individus (piscines, bijoux, ordinateurs). Les entreprises touristiques devront donc porter une attention particulière à la concurrence indirecte, aux produits et services qui ne sont pas du domaine touristique, mais qui peuvent répondre à une quête de l'exotisme chez soi. A ce chapitre, mentionnons que la différenciation doit se baser sur les ćéments déclencheurs et préalables à l'acte d'achat. Le degré d'expérience des touristes étant de plus en plus élevé, la reproduction ou la copie de l'offre touristique mènera à l'échec commercial.

Dans un troisième temps, les entreprises touristiques devront élaborer des produits et services touristiques qui tiennent compte du manque de temps et d'argent des individus. Le tourisme réceptif se présente alors comme une alternative permettant de répondre aux contraintes des gens. Toutefois, seules les entreprises locales originales pourront tirer leur épingle du jeu. Le tourisme réceptif sera une des alternatives, dans la mesure où il ne sera pas standardisé et qu'on saura développer, à partir d'une couleur locale, une expérience différente des destinations étrangères, mais également de a l'étrangeté * chez soi.

Finalement, on ne pourra contrer la quête d'exotisme chez soi disponible sur les réseaux de communication virtuelle. Les entreprises touristiques devront alors élaborer des produits et des services complémentaires virtuels qui conduiront les individus à passer du virtuel au réel. Par exemple, des entreprises pourraient utiliser une émission de télévision sur Internet afin de susciter les gens à venir vivre la différence sur les lieux touristiques. Cependant, il ne faudra pas se contenter des traditionnelles reproductions d'événements touristiques. Les entreprises devront plutôt démontrer le caractère unique de ces attractions et sites touristiques. La vente des produits et services touristiques sera alors plus complexe et débutera par une présence constante sur les réseaux de communication virtuelle. Ces réseaux devront donc être utilisés afin d'accroitre le pouvoir d'attraction des sites touristiques. L'erreur serait de simplement reproduire artificiellement les sites touristiques sur les réseaux de communication virtuels. Faire passer les individus du virtuel au réel, de la quête de l'exotisme de chez soi à̀ une quête de l'exotisme à l'étranger, représente le principal défi des prochaines années.

Lawrent Bourdean est professeur de marketing au Departement d'économie agroalimentaire et des sciences de la consommation de l'Université Laval. Pascale Marcotte est étudiante au programme de doctorat en sociologie à l'Université du Québec à Montréal.

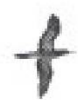

\section{NOTES}

1 Si une grande partic des touristes étrangers vont visiter un pays pour vivre une expérience culturelle différente et connaître différents modes de vie (Longwood, 1989), une autre partic, non moins importante, cherche avant tout le divertissement, le plaisir et la détente et ce, peu importe l'endroit où elle va.

2 Mill et Morrison (1985) affirment en effet que la décision de partir en voyage de plusieurs touristes s'appuie sur le désir d'impressionner l'entourage lors du retour.

3 L'identité culturelle peut se définir comme l'ensemble des traits et des éléments qui singularise une culture donnée et la valeur symbolique d'attachement que représentent des eléments che $z$ ceux qui la constituent (image de soi en tant que collectivité, genre de vie, vision du monde qui s'appuie autant sur une interprétation du vécu collectif que sur l'explication de projet de la communauté, adaptation au territoire, satisfaction des besoins du groupe, constitution d' un univers symbolique propre, partage des projets d'aveniry.

4 La notion de sentiment d'exotisme est utilisée selon le sens donné par Segalen (1986) lorsqu'il associe l'exotisme au different, at la connaissance de quelque chose qui n'est pas soi-même.
5 Le tourisme, tout comme certains musées. reçoit les mêmes critiques que celles faites à la culture de masse (Burgelin, 1967). Les traits dominants de la culture de masse semblent être, pour les tenants de la haute culture : sa facilité, son absence d'effort, tant pour le créteur que pour le consommateur. son uniformité, son appauvrissement culturel, son caractère amusant plutôt qu'enrichissant et son alienation.

6 Le lieu est défini par Augé (1992) comme étant identitaire, relationnel, historique. Il permet de concevoir l'espace comme un lieu de relations particulières et de positionner ses origines pour pouvoir se connaître. Le nonlieu est symptomatique de la perte des relations sociales (MacCannel, 1992).

\section{BIBLIOGRAPHIE}

Augé, Mare (1992), Non-lieux: Introduction à une anthropologie de la surmodernité, Paris. Éditions du Seuil, 149 p.

Benjamin, Walter (1971), Euvres II. Poésie et Révolution, Paris, Denoel-Gonthier, 292 p.

Burgelin, Olivier (1967), « Le tourisme jugé », Commanications, vol. 10.

Gourant, Pierre (1993), \& Le tourisme a l'aube de l'an 2000 : Quantité ou qualité ? \%, Téoros, vol. $12, \pi^{\circ} 2$, p. $22-26$.

Longwoods Travel USA (1991), Le marche américain des voyages d'agrément 1989 , pour Tourisme Canada, janvier, $89 \mathrm{p}$.

MacCannel, Dean (1992), Empty Meeting Grounds ; The Tourist Papers, Londres, Routledge, $346 \mathrm{p}$.

Mannell, R.C. et Iso-Athola, S.E. (1987), $\propto$ Psychological Nature of Leisure and Tourism Experience, $*$ Annals of Tourism Research, 14, p. 314-361.

Mill, R.C. et A.M. Morrison (1985), The Tourist System, Englewood Cliffs, NJ, Prentice-Hall.

Segalen, Victor (1986), Essai sur l'exotisme, whe esshétique du divers, Paris, Fata Morgana, 157 p. 